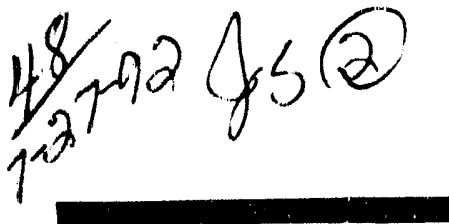

PPPL-2851

UC -427
PREPARED FOR THE U.S. DEPARTMENT OF ENERGY, UNDER CONTRACT DE-AC02-76-CHO-3073

PPPL-2851

\title{
A FULLY NONLINEAR CHARACTERISTIC METHOD FOR GYROKINETIC SIMULATION
}

BY

S.E. PARKER AND W.W. LEE

July, 1992
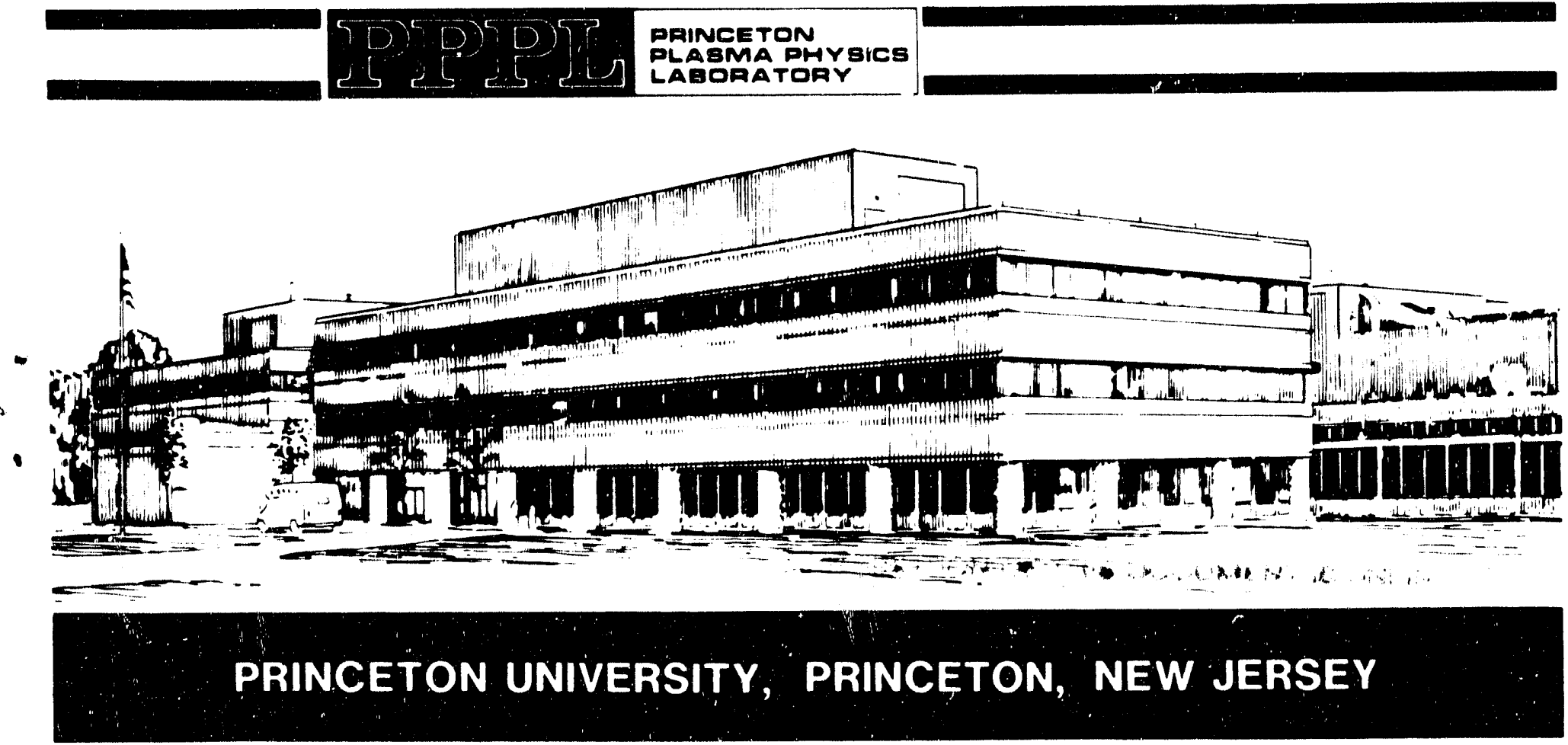
NOTICE

This report was prepared as an account of work sponsored by an agency of the United States Government. Neither the United States Government nor any agency thereof, nor any of their employees, makes any warranty, express or implied, or assumes any legal liability or responsibility for the accuracy, completeness, or usefulness of any information, apparatus, product, or process disclosed, or represents that its use would not infringe privately owned rights. Reference herein to any specific commercial produce, process, or service by trade name, trademark, manufacturer, or otherwise, does not necessarily constitute or imply its endorisement, recommendation, or favoring by the United States Government or any agency thereot. The views and opinions of authors expressed herein do not necessanily state or reflect those of the United States Government or any agency thereof.

NOTICE

This report has been reproduced directly from the best available copy.

Available to DOE and DOE contractors from the:

Office of Scientific and Technical Information

P.O. Box 62

Oak Ridge, TN 37831 ;

Prices available from (615) 576-8401.

Available to the public from the:

National Technical Information Service

U.S. Department of Commerce

5285 Port Royal Road

Springfield, Virginia 22161

$703-487-4650$ 


\title{
A Fully Nonlinear Characteristic Method for Gyrokinetic Simulation
}

\author{
S. E. Parker and W. W. Lee \\ Princeton Plasma Physics Laboratory, Princeton University \\ Princeton. N.J 08543
}

\begin{abstract}
We present a new scheme which evolves the perturbed part of the distribution function along a set of characteristics that solves the fully nonlinear gyrokinetic equations. This nonlinear characteristic method for particle simulation is an extension of the partially linear weighting scheme, ${ }^{1}$ and may be considered an improvement of existing $\delta f$ methods. ${ }^{2.3}$ Some of the features of this new method are: the ability to keep all the nonlinearities. particularly those associated with parallel acceieration: the loading of the physical equilibrium distribution function $f_{0}$ (e.g.. a Maxwellian), with or without the multiple spatial scale approximation: ${ }^{4}$ the use of a single set of trajectories for the particles: and also, the retention of the conservation properties of the original gyrokinetic system in the numerically converged limit (i.e., small $\Delta t$, small $\Delta . x$ and a large number of particles). Therefore, one can take advantage of the low noise property of the weighting scheme together with the quiet start techniques to simulate weak instabilities, with a substantially reduced number of particles than required for a conventional simulation. The new method is used to study a one dimensional drift wave model which isolates the parallel velocity nonlinearity. $I$ mode coupling calculation of the saturation mechanism is given, which is in good agreement with the simulation results and predicts a considerably lower saturation level then the estimate of Sagdeev and Galeev. ${ }^{5}$ Finally, we extend the nonlinear characteristic method to the electromagnetic gyrokinetic equations in general geometry.
\end{abstract}

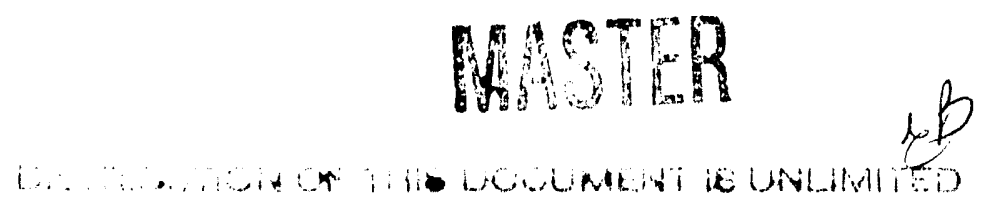




\section{Introduction}

An outstanding issue in gyrokinetic simulation. (and in particle-in-cell simulation in gerieral) is that often times it is necessary to use a rather large number of particles to resolve the physics of interest. The concern being that thermal fluctuations (or noise) and lack of sampling (or resolution) of the two to six dimensional phase space caused by a finite number of particles may obscure the physical process being modeled. For example. the broad-band density fluctuations existing in tokamak plasmas usually have an average level of a fraction of one percent or less. Thus, modeling such plasmas requires a very large number of particles for proper resolution. such that $N_{\text {tot }} \gg 1 /(\delta n / n)^{2} \approx 10^{6}{ }^{4}$ Early on it was recognized by Beyers and others that improvements in noise properties could be made by loading more uniform distributions or "quiet starts, ${ }^{" 6-8}$ and also by linearization techniques which track the perturbed quantities in terms of the equilibrium trajectories. ${ }^{9,10}$ More recently, Dimits and Lee ${ }^{1}$ developed a linear and a "partially linear" scheme by allowing the particle weights to evolve in time. In the linear scheme the particle weights follow the equilibrium trajectories. In the partially linearized scheme, the $E \times B$ nonlinearity is retained by adding this drift to the equilibrium (zeroth-order) orbit. There have been

other investigations ${ }^{2,3}$ into similar techniques often called $\delta f$ methods, but no schemes up to this point have reported actual simulation results where the parallel velocity nonlinearity is retained.

The nonlinear characteristic method presented here is similar to previous weighting schemes; ${ }^{1}$ however. the fully nonlinear trajectories are evolved and a consistent evolution equation is used for the particle weights. We begin by presenting the new method for the electrostatic slab case and the associated conservation properties of particle number, momentum, and total energy. We then present gyrokinetic simulations of a simpie one dimensional drift wave instability in a shearless slab. This model isolates $E_{\|} \partial_{v^{\prime} \|}$ of nonlinearity and allows us to study the associated nonlinear physics. A three wave mode coupling theory is presented which gives a saturation level of $e|\phi| / T_{e} \approx 5.5 \gamma^{2} /\left(k_{\|} v_{t e}\right)^{2}$ which is much lower than the calculation of Sagdeev and Galeev, ${ }^{5} e|\phi| / T_{e} \approx \frac{1}{4}\left(k_{\perp} \rho_{s}\right)^{2}$. There is good agreement between theory and simulation in terms of linear frequency, growth rate, and 
nonlinear saturation. The conservation properties of the simulation plasma have also been investigated. It is found that the conservation of number density, momentum and energy in the nonlinear stages of the simulation can be achieved only if we use a sufficiently large number of particles, small timesteps. and a small grid cell size. Finally, we discuss the application of the nonlinear characteristic method to the general electromagnet ic (nomuniform equilibrium magnetic field) gyrokinetic equations.

\section{Nonlinear Characteristic Method}

As mentioned earlier, devising a scheme where only the perturbed part of the distribution function is evolved has been previously investigated. ${ }^{1-3}$ It was recognized that, by evolving only the perturbed part of the distribution $\delta f$, one could remove the initial noise associated with the equilibrium $f_{0}$ due to the use of a finite number of particles. ${ }^{6}$ The scheme we present here is similar to the previously proposed $\delta f$ methods, ${ }^{2,3}$ but we take into account the discrete representation of the characteristics in a similar fashion as was done for the linear and partially linearized schemes. ${ }^{1}$

Let us begin by writing the distribution function $f\left(\mathbf{R}, v^{\prime} \|, \mu\right)$ in the familiar way as $f=f_{0}+\delta f$. where $f_{0}$ is the equilibrium background distribution and is independent of time, and $\delta f$ is the perturbed time dependent part of the distribution. For $\left(k_{\perp} \rho_{i}\right)^{2} \ll 1$. the gyrokinetic equation for $\delta f$ in the electrostatic slab limit is

$$
\partial_{t} \delta f+\mathrm{v}_{E} \cdot \nabla \delta f+v_{\|} \nabla_{\|} \delta f+\frac{q}{m} E_{\|} \partial_{v_{\|}} \delta f=-\mathbf{v}_{E} \cdot \nabla f_{0}-\frac{q}{m} E_{\|} \partial_{v_{\|}} f_{0} .
$$

where $\rho_{i} \equiv v_{t i} / \Omega_{i}$ is the ion gyroradius, $\mathbf{v}_{E} \equiv c \mathbf{E} / B \times \hat{\mathbf{b}}$ is the $\mathbf{E} \times \mathbf{B}$ drift. The corresponding gyrokinetic Poisson equation in the small $\left(k_{\perp} \rho_{i}\right)^{2}$ limit is

$$
\left(\rho_{s} / \lambda_{D}\right)^{2} \nabla_{\perp}^{2} \emptyset=-4 \pi e\left(n_{i}-n_{e}\right)
$$

where $\rho_{s} \equiv \sqrt{\tau} \rho_{i}, \tau \equiv T_{e} / T_{l}, \lambda_{D} \equiv \sqrt{T_{e} /\left(4 \pi n_{0} e^{2}\right)}$ is the Debye length. and

$$
n=\int \delta f d v_{\|} d \mu
$$


The characteristics (or trajectories) of Eq. (1) are

$$
\begin{aligned}
& \dot{v}_{\|}=\frac{q}{m} E_{\|}, \\
& \dot{\mathrm{R}}=\|_{\|} \dot{\mathrm{b}}+\mathrm{v}_{E},
\end{aligned}
$$

and along these characteristics $\delta f$ is changing because the right hand side of Eq. $(1)$ is nonzero (note that $\dot{\mu}=0$ here). The evolution equation for $\delta f$ along the trajectories is

$$
\dot{\phi} f=-\mathrm{v}_{E} \cdot \nabla f_{0}-\frac{q}{m} E_{\|} \dot{\partial}_{v^{\prime} \|} f_{0} .
$$

Now, we simply need to solve Eqs. (4)-(6). At first glance, one might consider loading a large number of characteristics (or particles). each having it's own $\delta f$. evolve the system in time and then weight the $\delta f$ 's to the grid to obtain field quantities. However, one needs to examine carefully how $\delta f$ is being numerically represented and whether Eq. (1) or $(6)$ is indeed being solved correctly.

In a similar fashion as the partially linear weighting scheme, ${ }^{+}$except now the fully nonlinear trajectories are evolved, $\delta f$ in the simulation is represented as

$$
\partial f\left(\mathbf{R} . v_{\|} \cdot \mu \cdot t\right)=\sum_{i=1}^{N} \omega_{i} s^{\prime}\left(\mathbf{R}-\mathbf{R}_{i}\right) \delta\left(v_{\|}-v_{\|_{2}}\right) \delta\left(\mu-\mu_{1}\right) .
$$

where.$V$ is the total number of particles and $S$ is the particle shape function. $u_{i}$ is the particle weight and can be interpreted as the $\delta f / f$ associated with the particle $i$. as will be shown below. $S(u)=\lambda(u)$ is used for present discussion (i.e.. we assume the particle shape function has the same properties as the delta function). This is equivalent to assuming that all quantities have a small spatial (or long wavelength) variation compared to the particle size. One can then use relations such as the following

$$
\begin{aligned}
\sum_{i} f\left(\mathbf{R}_{1}\right) \cdot S\left(\mathbf{R}-\mathbf{R}_{i}\right) & =\sum_{i}\left(f(\mathbf{R})-\left(\mathbf{R}-\mathbf{R}_{i}\right) \cdot \nabla f(\mathbf{R})+\cdots\right] S\left(\mathbf{R}-\mathbf{R}_{i}\right) \\
& \approx f(\mathbf{R}) \sum_{i} S\left(\mathbf{R}-\mathbf{R}_{i}\right) .
\end{aligned}
$$

Substituting Eq. (i) into Eq. (1) we obtain the following relation.

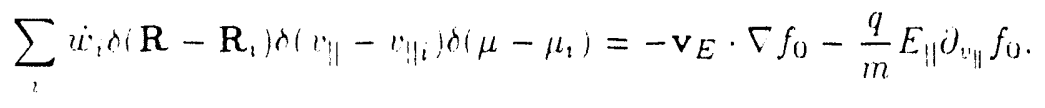


It should be noted here that Eq. (10) and Eq. (6) are quite different. Namely, the avolution of $\dot{w}$ from Eq. (10) is not the same as assuming $\dot{w}=\dot{\delta} f$ and using Eq. (f) to evolve $w$ along it's trajectory. We will elaborate this point later. Next, we assume that the particle distribution can be represented as a smooth analytical function

$$
g\left(\mathbf{R}, v_{\|}, \mu . t\right) \approx \sum_{i} \delta\left(\mathbf{R}-\mathbf{R}_{i}\right) \delta\left(v_{\|}-v_{\| i}\right) \delta\left(\mu-\mu_{i}\right) .
$$

( $g$ could be equal to the physical distribution $f$. but this is not necessary up to this point.) From Eqs. (10) and (11). we obtain

$$
\dot{w}_{i}=-\left[\mathbf{v}_{E} \cdot \frac{\nabla f_{0}}{g}+\frac{q}{m} E_{\|} \frac{\partial_{v_{\|}} f_{0}}{g}\right]_{\mathbf{R}=\mathbf{R}_{\imath}, v_{\|}=v_{\| r}, \mu=\mu_{1}, t} .
$$

One way to solve $\mathrm{Eq} .(12)$ is to note that the function $g$ satisfies the full nonlinear gyrokinetic equation so that $g$ is constant along a trajectory. One then can use the relation $g\left(\mathbf{R}_{i}, v_{\| i}, \mu_{i}, t\right)=g\left[\mathbf{R}_{i}(t=0), v_{\|_{i}}(t=0), \mu_{i}, t=0\right]$. Since the initial loading is arbitrary, one can, for example. load a uniform distribution $g(t=0)=$ const. independent of the phase space variables, which seems to be the approach proposed by Kotschenruether. ${ }^{3}$

However. the equilibrium $\int_{0}$ with a nonuniform loading in the velocity space (with or without spatial inhomogeneity) is preferred, because one wants more phase space resolution (i.e. particles) where the wave is in resonance with the distribution function $\left(\omega / k_{\|}=v_{\|}\right)$. Typically, for low frequency microinstabilities, the resonant velocities are smaller than the thermal velocity of the species. Thus, a nonuniform loading (e.g., the Maxwellian distribution) in velocity space is much more desirable than a uniform one. One problem associated with the nonuniform loading for the existing $\delta f$ schemes is that additional particle arravs (or calculations) are needed to keep track of $g_{i}(t=0)$. Moreover, numerical difficulties may arise from the denominators of $\mathrm{Eq}$. (12) for particles with small values of $g_{i}(t=0)$, i.e.. those initially located in the tail of the distribution. These are the potential drawbacks for using Eq. (12) to evaluate $w_{i}$, along with $g_{i}(t=0)$.

We now present the nonlinear characteristic method, which avoids these problems. One loads the particles with the equilibrium distribution $g(t=0)=f_{0}$ (as above. $g$ is the particle distribution) in the same manner as in the usual particle simulation. Then we 
assume $y=f=\int_{0}+\delta f$ throughout the simulation. Since $\frac{1}{f}=\frac{1}{f_{0}}\left(1-\frac{\delta f}{f}\right)$. from Eq. (12), we arrive at

$$
\dot{u}_{i}=-\left[\left(\mathbf{v}_{E} \cdot \frac{\nabla f_{0}}{f_{0}}+\frac{q}{m} E_{\|} \frac{\partial_{1^{\prime} \|} f_{0}}{f_{0}}\right)\left(1-\frac{\delta f}{f}\right)\right]_{\mathbf{x}=\mathbf{x}_{1}, v_{\|}=v_{\|,}, \mu=\mu_{1}, t} .
$$

To determine $\delta f / f$ in Eq. (13), we note the following

$$
\begin{aligned}
\delta f & \approx \frac{\delta f}{f} \sum_{i} \delta\left(\mathbf{R}-\mathbf{R}_{i}\right) \delta\left(v_{\|}-v_{\| i}\right) \delta\left(\mu-\mu_{i}\right) \\
& =\sum_{i} \delta\left(\mathbf{R}-\mathbf{R}_{i}\right) \delta\left(v_{\|}-v_{\|}\right) \delta\left(\mu-\mu_{i}\right)\left[\frac{\delta f}{f}\right]_{\mathbf{x}=\mathbf{x}_{1}, v_{\|}=v_{\|,}, \mu=\mu_{i}, t} .
\end{aligned}
$$

The "approximately equal to" sign was used again because we assumed that $f$ and $\delta f$ were smooth functions, as was done in Eq. (11). From Eqs. ( 7 ) and (15) we see that $w_{i}=[\delta f / f]_{i}$, and, hence, we can substitute this into Eq. (13) to obtain

$$
\dot{w}_{i}=-\left(1-w_{i}\right)\left[\left(\mathbf{v}_{E} \cdot \frac{\nabla f_{0}}{f_{0}}+\frac{q}{m} E_{\|} \frac{\partial_{v_{\|}} f_{0}}{f_{0}}\right)\right]_{\mathbf{x}=\mathbf{x}_{1}, v_{\|}=v_{\|_{0}}, \mu=\mu_{1}, t} .
$$

Equation (16) along with Eqs. (4), (5) and ( 7 ) are the crux of the new nonlinear characteristic method [as usual, one uses Eqs. (2) and (3) to complete the loop]. Besides the nonlinearities appearing as perturbations to the zeroth-order trajectories $\left[E_{\|}\right.$and $\mathbf{v}_{E}$ in Eqs. $(4)$ and $(5)]$, there is the additional factor $\left(1-w_{i}\right)$ in the evolution of the equation for the weights in Eq. (16). This factor, which is essential to the nonlinear physics and the conservation properties of the simulation plasma, describes the difference between $f_{0}(t=0)$ and $f_{0}(t)$, as indicated by Eq. (12).

Let us now discuss the conservation of the total particle number, momentum and total energy associated with the new nonlinear weighting scheme. First, we examine the change in "the number of particles" by taking the zeroth velocity moment of Eq. (1). Using Eq. $(T)$ and assuming that the sum of the $w_{i}$ 's is nearly zero initially, we then obtain

$$
\sum_{i} w_{i}(t)=0
$$

The momentum conservation can be derived by taking the first velocity moment of Eq. (1),

$$
\partial_{t}\left\{\int v_{\|} \delta f d \mathbf{R} d \mu d v_{\|}\right\}-\frac{q}{m}\left\{\int n E_{\|} d \mathbf{R}\right\}=0 .
$$


which, along with Eqs. (2) and ( 7$)$, then gives

$$
\sum_{\alpha} m_{\alpha} \sum_{i} v_{\| \alpha i} w_{\alpha i}=0
$$

where $n$ is given by Eq. (3) and $\alpha$ denotes species. To calculate the change in energy, ${ }^{4}$ we take the second velocity moments of Eq. (1) for both the ions and the electrons and substituting them into Eq. (2). Again, if we take the particle weights and field to be zero at $t=0$, then the simulation should conserve energy, i.e.,

$$
\sum_{\alpha} \frac{m_{\alpha}}{2} \sum_{i}\left(\mu_{\alpha i}+v_{\| \alpha i}^{2}\right) w_{\alpha i}+\left(\frac{\rho_{s}}{\lambda_{D}}\right)^{2} \frac{1}{8 \pi} \int\left|\nabla_{\perp} \phi\right|^{2} d \mathbf{R}=0
$$

We expect Eqs. (1i)-(20) to hold for a simulation plasma in the limit of large number of particles and fine space and time resolution. In the next section, we will examine the validity of these conservation properties in a simulation using a finite number of particles. This is an important exercise which serves as a test for both the formal correctness as well as the practicality of the proposed scheme. In other words, we want to examine the scheme to see if it is a useful alternative to conventional particle methods.

\section{One Dimensional Drift Wave Simulation}

We now present simulation results using the new method checking linear and nonlinear physics as well as the conservation properties. For simplicity, a one dimensional drift wave problem is chosen as an example here. In this one dimensional model the $E \times B$ nonlinearity is not present, so the $E_{\|} \partial_{\nu_{\|}} \delta f$ nonlinearity is the only mechanism for saturation. This is the term which has been neglected in the previous scheme ${ }^{1}$ on the assumption that, in the more realistic two and three dimensional geometries, it is the the $E \times B$ nonlinearity which is the dominant saturation mechanism. However, as suggested in Ref. 10 and 11 , the $E_{\|} \partial_{v_{\|}} \delta f$ nonlinearity may be relevant in determining the steady-state transport for gradient-driven microinstabilities.

In this problem the one spatial dimension $y$ is perpendicular to the spatial gradients, which are in the $x$ direction, and is almost perpendicular to the magnetic field, $\hat{b}=\dot{z}+\theta \hat{\mathbf{y}}$. 
Here, both $x$ and $z$ are ignorable coordinates for the perturbation quantities. The one dimensional version of Eq. (1) for both the ions and the electrons is

$$
\partial_{t} \delta f+\theta v^{\prime} \| \partial_{y} \delta f-\alpha \theta \partial_{y} \phi \partial_{v_{\|}} \delta f=-\kappa \partial_{y} \phi f_{0}+\alpha \theta \partial_{y}, \frac{v^{\prime} \|_{\|}^{2}}{v_{t}^{2}} f_{0},
$$

where $\alpha=\left(1,-m_{i} / m_{e}\right)$ for the ions and the electrons, respectively; and we use the dimensionless gyrokinetic units of $y / \rho_{s}-y, \Omega_{i} t \rightarrow t, v_{\|} / c_{s} \rightarrow v_{\|}$, and $e \phi / T_{e} \rightarrow \phi$. For this problem, we use $\kappa=-\partial_{x} \ln n_{0}$ and have assumed a Maxwellian equilibrium. The gyrokinetic Poisson equation or the "quasineutrality" condition in one dimension is

$$
\partial_{y y} \phi+\delta n_{i}=\delta n_{e}
$$

We solve these equations with a one dimensional gyrokinetic simulation using the new method explained in Sec. 2. In addition, a quiet start technique employing Fibonacci numbers ${ }^{8}$ has been used. In order to further minimize the noise, we have also used a cutoff scheme ${ }^{1}$ of

$$
w_{i}=\phi\left(x_{i}\right)
$$

for the fast particle with $v_{\| i} \gg \omega / k_{\|} . \phi_{m}$ is initially perturbed, where $m$ is the Fourier harmonics of interest. Since our purpose is to study the nonlinear electron dynamics, we have also linearized the ion motion in the simulation by discarding the $\partial_{v_{\|}} \delta f$ term in $\mathrm{Eq}$. (21), which is accomplished in the simulation by letting $v_{\|}=0$ for $\mathrm{Eq}$. (4) and dropping the $\left(1-w_{i}\right)$ correction in Eq. (16).

The first run shown has the following parameters in the gyrokinetic units: $T_{e} / T_{i}=$ $1, m_{i} / m_{e}=1837$. the magnetic field tilt $\theta=0.01$, the particle size is one $\left(=\rho_{s}\right)$, the timestep is $\Delta t=1 . \kappa_{n}=0.2$, the system size is $L=16 \Delta x$, the grid size is $\Delta x=0.5$, and the total number of particles is $V_{\text {tot }}=f_{16}=987$, where $f_{16}$ denotes the sixteenth Fibonacci number. With this choice of parameters the dominant unstable mode is the $n=1$ hurmonic or $k=2 \pi / L \simeq 0.8$ mode. Figure $1(\mathrm{a})$ shows the the time evolution of the electrostatic potential for $n=1$ Fourier mode. The real part is the solid line and the imaginary part is the dashed line. The mode frequency averaged over both the linear and nonlinear parts of the evolution is $\omega \simeq 0.075$. Figure $1(\mathrm{~b})$ gives the logarithmic change of the 
amplitude as a function of time for this mode. A clean linear growth followed by a sudden nonlinear saturation is clearly visible. The measured linear growth rate is $\gamma \simeq 0.012$. and the saturation amplitude is $\phi \simeq 1.1 \%$. Figure $2($ a) shows the spatially averaged perturbation $\delta f_{e 0}(\equiv\langle\delta f\rangle)$ at $t=500$ and gives the resonant point at $v_{\|}=0.2 v_{t e}$. Integrating $\delta \int_{e 0}$ in $v_{\|}$, we obtain $\left|\sum_{i} w_{i} / N_{t o t}\right| \simeq 1.3 \times 10^{-4}$ at $t=500$; thus, the particle conservation deviates from Eq. (17). This discrepancy, which increases by a factor of 2.5 at the end of the run, has no effect on the instability, it is however, a measure of accuracy of the simulation. Another interesting aspect of the simulation is that, while we are solving Eq. (21), we also follow the evolution of the equation $\dot{f}=0$ with the same set of equations of motion and

$$
f\left(y, v_{\|}, t\right)=\sum_{i=1}^{N} S\left(y-y_{i}\right) \delta\left(v_{\|}-v_{\| i}\right) .
$$

The total distribution function $f$ at $t=500$ is shown in Fig. 2(b). Its jaggedness, compounded by the fact that $\delta f_{e 0}$ is nou at all discernible, would make it impossible to use this information for conservation property diagnostics, let alone for the field solve.

Because of the use of the linear ion response, the momentum conservation given by Eq. (18) cannot be satisfied in the simulation. However, we can use Eq. (19) to check a similar property. In the one dimensional system, it becomes ${ }^{12}$

$$
\frac{d}{d t}\left\langle\int v_{\|} \delta \int_{e} d v_{\|}\right\rangle+\frac{m_{i}}{m_{e}} \theta\left\langle\Gamma_{e}\right\rangle=0
$$

where $\Gamma_{e} \equiv-\partial_{y} \phi n_{e}$ is the particle flux, and $\langle\cdots\rangle$ denotes spatial average. Hence, we obtain a relationship between the rate of change of the momentum and the particle flux in the simulation. which is plotted in Fig. 3(a). Here, a frequency filter has been used to smooth the data for $d p_{e} / d t$ and the normalization constant $n_{0}$ is the average number density. Apparently, numerical noise for the flux is quite substantial and, again. there is a discrepancy of $O\left(10^{-4}\right)$ between these two quantities. From Eq. (20), the energy conservation becomes,

$$
\left\langle\int \delta f v_{\|}^{2} d v_{\|}\right\rangle / n_{0} v_{t} e^{2}+\left\langle\left|\partial_{y} \phi\right|^{2}\right\rangle=0 .
$$

This is shown in Fig. 3(b). Again, the results are quite noisy and the difference between the kinetic energy and the field energy is also of $O\left(10^{-4}\right)$. However, considering the small 
number of particles used in the simulation, these results are actually quite good. We remind the reader that this was accomplished through the use of: 1) nonlinear weighting scheme. 2) the quiet start technique, and 3) the nonrandom initial perturbation.

Nevertheless, the errors in the conservation properties after nonlinear saturation is troublesome, since our ultimate goal is to use the scheme to study long-time steady-state phenomena. To improve upon these results, we have carried out a series of runs and found that a substantial increase in numerical accuracy is needed. To illustrate this point, we now present a run with $\Delta t=0.2, V_{t o t}=46,368$ particles on a 6.4 -grid system using a particle size of $\rho_{s} / 2$. All the other parameters remain the same. Figures $4(\mathrm{a})$ and (b) show the mode history. Comparing with the previous case, the mode frequency (averaged over both the linear and nonlinear parts of the evolution ) increases slightly to $\omega \simeq 0.088$, and the saturation level decreases slightly to $\phi \simeq 1 \%$, while the growth rate remains the same at $\gamma \simeq 0.012$. One important difference is that the numerical (including noise) errors remain small in the nonlinear stage when the particles are trapped and executing bounce motion. This characteristic is clearly manifested in the diagnostics for the perturbed distribution function $\delta f_{e 0}$ and $f$ in Figs $5(a)$ and (b). However, for the perturbed distribution, which is also measured at $t=500$, the asymmetry in the velocity space still remains, and the corresponding discrepancy for the total particle number, $\left|\sum_{i} w_{i} / N_{t o t}\right|$, averages to about $1.35 \times 10^{-4}$, which is similar to the previous course simulation. However, the overall smoothness of the distributions in Figs. 5(a) and (b) come from the increase in numerical accuracy. Interest. ingly, even with this type of accuracy, the perturbation of $\delta \int_{e 0}$ is still not quite discernible in the total $f$ diagnostic Fig. $5(b)$. This is because the perturbation is only a factor of two above the thermal fluctuation level of $\phi=1 / \sqrt{N_{\text {tot }}} k \simeq 0.6 \%{ }^{4}$ Thus, following from Ref. 1 , one can surmise that a total $f$ simulation. even with this many particles, would not give as clean a result as the new scheme. This point cannot be verified, however, because there is no available scileme to solve the equation of the form, $\dot{f}=-\kappa \dot{\partial}_{y} \phi f_{0}$. The corresponding flux and energy diagnostics for this case are shown in Figs. 6(a) and (b). As we can see. the conservation of both these quantities are near perfect. The implication here seems to be that one still has to use a very large number of particles with enoligh spatial and time 
resolution to obtain reasonable conservation properties. On the other hand, a conventional particle simulation would need even more. However, if one is only interested in linear growth and nonlinear saturation. the present scheme represents a substantial savings in computing resources.

\section{Nonlinear Saturation}

The nonlinear saturation of the most unstable modes (e.g., $n= \pm 1$ in the simulation results presented above) is due to the parallel velocity nonlinearity and is not caused by $E \times B$ advection. It is commonly assumed that the the $E \times B$ nonlinearity, which is absent in the simple one dimensional model, is the dominant nonlinearity for the saturation of the drift waves in the (more realistic) higher dimensional models. However, the parallel nonlinearity does play a role in the saturation as was shown in Ref. 11. The simulation results in Sec. 3 indicate that the satiration level is comparable to the $E \times B$ saturation level $\epsilon|\phi| / T_{e} \sim \gamma /\left(k_{\perp}^{2} c_{s} \rho_{s}\right)\left(\right.$ assuming $\left.k_{x} \sim k_{y}\right),{ }^{11}$ and is much lower then $e|\phi| / T_{e} \sim \frac{1}{4}\left(k_{\perp} \rho_{s}\right)^{2}$, as predicted by Sagdeev and Galeev. ${ }^{5}$ Also, in a tokamak geometry, drift cype modes are elongated in the radial direction $\left(k_{r} \ll k_{\theta}\right)$, at least in the linear phase, which should reduce the effect of the $E \times B$ on saturation. ${ }^{13-15}$ In addition, the parallel nonlinearity may be important in determining the steady-state transport caused by microturbulence. ${ }^{16}$ which will not be discussed here. The one dimensional model allows us to isolate the parallel nonlinearity and study the associated physics.

In this section, we consider a simple case of three-wave coupling between the two fastest growing modes $(n= \pm 1)$ and $\delta f_{0}$ for the electrons. The saturation takes place when the electron electron distribution is steepened at the resonance point $k_{\|} v_{\|}=\omega$. The saturation amplitude of the potential due to the $E_{\|} \partial_{1, y} \delta \int$ nonlinearity is calculated using a quasilinear estimate whirh is similar to the calculation for saturation of drift waves due to the $E \times B$ nonlinearity given in Ref. 11. In the following, we use the subscript "1" to label the fastest growing mode and its complex conjugate with $k=2 \pi n / L$. where $L$ is the system length. and $n$ is the Fourier mode number. [Note that $\delta f_{1}(k)=\delta f_{1}^{*}(-k)$ ]. The governing drift-kinetic 
electron equations are

$$
\begin{aligned}
\partial_{t} \delta f_{1}+k_{\|} v_{\|} \partial_{y} \delta f_{1}+i\left(\omega_{*}-k_{\|} v_{\|}\right) f_{0}+i \frac{m_{i}}{m_{e}} k_{\|} \phi_{1} \partial_{v_{\|}} \delta f_{0} & =0 \\
\partial_{t} \delta f_{0}-2 \frac{m_{i}}{m_{e}} k_{\|} \phi_{1} \partial_{v_{\|}} \operatorname{Im}\left(\phi_{1} \delta f_{1}^{*}\right) & =0
\end{aligned}
$$

where $\omega_{*}=\kappa k$ (in the gyrokinetic units). $f_{0}$ is the background Maxewellian and $\delta f_{0}$ is the nonlinear change of the background due to mode coupling. The perturbed electron density is $\delta n_{e 1}=\int \delta f_{1} d v_{\|}$. For the ions, we assume a fluid response since $\left|\omega / k_{\|}\right| \gg v_{t i}$, and the continuity equation for ion density becornes

$$
\partial_{t} \delta n_{i 1}+i \omega_{\star} \delta n_{i 1}=0
$$

Equations (22) and (27)-(29) could be solved using the Vlasov (Eulerian) simulation and should give the same results as the particle simulation in the previous section. However, to obtain an analytic estimate, we assume the dependence of $e^{-i \omega t}$ for $\delta f_{1}, n_{i 1}$, and $\phi_{1}$, where $\omega=\omega_{r}+i \gamma$ and also $\left|\omega_{r} / \gamma\right| \gg 1$. The perturbation can then be expressed as

$$
\delta f_{1}=\left\{f_{0}-\frac{\left(\omega_{*}-\omega_{r}\right)}{\left(k_{\|} v_{\|}-\omega\right)} f_{0}-\frac{m_{i}}{m_{e}} \frac{k_{\|}}{\left(k_{\|} v_{\|}-\omega\right)} \partial_{v_{\|}} \delta f_{0}\right\} \phi_{1} .
$$

Assuming $\gamma$ small, we can use the following relation

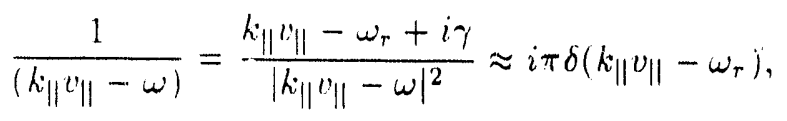

to obtain the electron density response as

$$
\delta n_{e, 1}=\int_{-\infty}^{+\infty} \delta f_{1} d v_{\|}=\left\{1-i \sqrt{\frac{\pi}{2}} \frac{1}{k_{\|} v_{t e}}\left(\omega_{*}-\omega_{\tau}\right)-\frac{m_{i}}{m_{e}} \int_{-\infty}^{+\infty} \frac{k_{\|}}{\left(k_{\|} v_{\|}-\omega\right)} \partial_{v_{\|}} \delta f_{0} d v_{\|}\right\} \phi_{1} .
$$

From which we arrive at the following nonlinear dispersion relation

$$
1+k^{2}-\frac{\omega_{*}}{\omega}-i \sqrt{\frac{\pi}{2}} \frac{1}{k_{\| !} v_{t e}}\left(\omega_{*}-\omega_{r}\right)-\frac{m_{i}}{m_{e}} \int_{-\infty}^{+\infty} \frac{k_{\|}}{\left(k_{\|} v_{\|}-\omega\right)} \partial_{v_{\|}} \delta f_{0} d v_{\|}=0
$$

Neglecting the nonlinear term (last term on the right), we obtain the familiar linear results ${ }^{11}$

$$
\begin{aligned}
\omega_{\tau}=\omega_{l} & \equiv \frac{\omega_{*}}{1+k^{2}}, \\
y=\gamma_{l} & \equiv \sqrt{\frac{\pi}{2}} \frac{1}{\left(k_{\| l} \omega_{t e}\right)} \frac{\omega_{l}}{\left(1+k^{2}\right)}\left(\omega_{*}-\omega_{l}\right) .
\end{aligned}
$$


The predicted linear frequency and growth rate for the simulation are: $\omega_{l}=0.09 i f$ and $\gamma_{l}=0.0134$, which are very close to the results shown in Figs. 1 and 4 . From the linear response for $\delta f_{1}$, the nonlinear response of the background can be estimated as

$$
\delta f_{0}=\pi \frac{m_{i}}{m_{e}} \frac{k_{\|}}{\gamma}\left|\phi_{1}\right|^{2}\left(\omega_{*}-\omega_{l}\right) \partial_{v_{\|}}\left\{\delta\left(k_{\|} v_{\|}-\omega_{l}\right) f_{0}\left(v_{\|}\right)\right\} .
$$

Substituting Eq. (36) into Eq. (33), we obtain the nonlinear dispersion relation in terms of the amplitude of $\phi_{1}$

$$
1+k^{2}-\frac{\omega_{*}}{\omega}-i \sqrt{\frac{\pi}{2}} \frac{1}{k_{\|} v_{t e}}\left(\omega_{*}-\omega_{l}\right)\left\{1-2 \frac{k_{\|}^{4}}{\gamma_{l}^{4}}\left(\frac{m_{i}}{m_{e}}\right)^{2}\left|\phi_{1}\right|^{2}\right\}=0 .
$$

From this equation, again assuming $|\gamma / \omega| \ll 1$, we obtain the same real frequency $\omega_{r}=\omega_{l}$, but the the quasilinear value for the growth rate becomes

$$
\gamma=\gamma_{l}\left\{1-2 \frac{\left(k_{\|} v_{t e}\right)^{4}}{\gamma_{l}^{4}}\left|\phi_{1}\right|^{2}\right\} .
$$

At saturation $\gamma=0$, the saturation level of the potential can be expressed as

$$
\left|\phi_{1}\right|=\frac{1}{\sqrt{2}} \frac{\gamma_{l}^{2}}{\left(k_{\|} v_{t e}\right)^{2}} .
$$

Using the simulation parameters of $k_{\|}=\theta 2 \pi / L=0.00785, m_{i} / m_{e}=1837$, and $\gamma_{l}=0.0134$. the predicted saturation amplitude is $\left|\phi_{1}\right|=0.11 \%$, which is an order of magnitude smaller than the level obtained from the simulation results shown in Figs. 1 and 4 . Thus, the effect of parallel nonlinearity on the saturation is overly estimated by the above approximation.

Next, to improve on this first estimate, we use the original form of the resonant denominator, Eq. (31), and also use the full nonlinear $\delta f_{1}$ to calculate $\delta f_{0}$, i.e...

$$
\delta f_{0}=\frac{m_{i}}{m_{e}} k_{\|}\left|\phi_{1}\right|^{2}\left(\omega_{*}-\omega_{r}\right) \partial_{v_{\|}}\left\{\frac{f_{0}}{\left|k_{\|} v_{\|}-\omega_{r}\right|^{2}}\left[1+\frac{m_{i}}{m_{e}} \frac{k_{\|}}{\left(\omega_{*}-\omega_{l}\right)} \frac{\partial_{v_{\|}} \delta f_{0}}{f_{0}}\right]\right\},
$$

If the quasilinear approximation is valid, this second order ordinary differential equation could be solved to obtain $\delta f_{0}$, which in turn could be used in Eq. (33) to obtain an accurate prediction of the saturation level. The extra term included in Eq. (40) accounts for the effect of parallel trapping on saturation. To obtain an simpler estimate, we begin by using the $\delta$-function relation, Eq. (31), in Eq. (40) to obtain

$$
\delta f_{0}=\left.(1-\alpha) f_{0}\right|_{\nu^{\prime} \|=\omega_{r} / k_{\|}} \frac{m_{i}}{m_{e}} k_{\|}\left|\phi_{1}\right|^{2}\left(\omega_{*}-\omega_{l}\right) \partial_{\nu_{\|}}\left|k_{\|} v_{\|}-\omega\right|^{-2},
$$


where $\alpha=-\left.\frac{m_{1}}{m_{\bullet}} \frac{k_{\|}}{\left(\omega_{*}-w_{l}\right)} \frac{\partial_{\nu \|^{\delta} f_{0}}}{f_{0}}\right|_{v_{\|}=\omega_{r} / k_{\|}}$. Then, substituting Eq. (41) in Eq. (33), assuming $\left|\omega / k_{\|}\right| \ll v_{t e}$, and integrating, we obtain the following relation for the saturation amplitude

$$
\left|\phi_{1}\right|=\frac{2}{\sqrt{1-\alpha}} \frac{\gamma_{i}^{2}}{\left(k_{\|} v_{t e}\right)^{2}}
$$

Assuming a lirear response for $\delta f_{1}$ as was done in deriving Eq. (39), so that $\alpha=0$, we obtain $\left|\phi_{1}\right|=0.32 \%$, which is in better agreement with the simulation results than Eq. (39), but it still too low.

An estimate of $\alpha$ can be made by taking the derivative of Eq. (40) with respect to $v_{\|}$, and evaluate at $v_{\|}=\omega_{r} / k_{\|}$to obtain

$$
\begin{aligned}
\left.\partial_{v_{\|}} \delta f_{0}\right|_{v_{\|}=\omega_{r} / k_{\|}}= & \frac{m_{i}}{m_{e}} k_{\|}\left|\phi_{1}\right|^{2}\left\{\left(\omega_{\kappa}-\omega_{l}\right) f_{0}\left(v_{\|}\right) \partial_{v_{\|}}^{2}\left|k_{\|} v_{\|}-\omega_{r}\right|^{-2}\right. \\
& \left.+\frac{m_{i}}{m_{e}} k_{\|}\left[\left(\partial_{v_{\|}} \delta f_{0}\right) \partial_{v_{\|}}^{2}+\left(\partial_{v_{\|}}^{3} \delta f_{0}\right)\right]\left|k_{\|} v_{\|}-\omega_{r}\right|^{-2}\right\}_{\nu_{\|}=\omega_{r} / k_{\|}},
\end{aligned}
$$

where we have neglected derivatives of $f_{0}\left(v_{\|}\right)$because it is slowly varying compared to $\left|k_{\|} v_{\|}-\omega_{r}\right|^{-2}$. We have also neglected $\partial_{v_{\|}}^{2} \delta f_{0}$ terms, by assuming $\delta f_{0}$ has the following form

$$
\delta f_{0}=C(t) v_{\|} \exp \left[-\frac{1}{2} \frac{v_{\|}^{2}}{\Delta v_{\|}^{2}(t)}\right],
$$

where $C$ and $\Delta v_{\|}$are independent of $v_{\|}$, and $\Delta v_{\|} \approx \sqrt{2\left|\phi_{1}(t)\right| \frac{m_{1}}{m_{e}}}$ is the width of the trapping region [see Fig. 5(a)]. From Eq. (44), one can show that $\left.\partial_{v_{\|}}^{2} \delta f_{0}\right|_{v_{\|}=\omega_{r} / k_{\|}} \simeq 0$. In addition. we can use the relationship of $\partial_{v_{\|}}^{3} \delta f_{0} \simeq-\frac{3}{\Delta v_{\|}^{2}} \partial_{v_{\|}} \delta f_{0}$, to obtain an equation for $\alpha$, i.e.,

$$
\alpha=\frac{2 z^{2}}{2 z^{2}+\frac{3}{2} z+1},
$$

where $z=\frac{\left(v_{\mathrm{te}} k_{\|}\right)^{2}}{\gamma_{l}^{2}}\left|\phi_{1}\right|$. We can now substitute this approximate value of $\alpha$ into Eq. (42) to obtain the following cubic equation

$$
\frac{3}{8} z^{3}-\frac{7}{4} z^{2}-\frac{3}{2} z-1=0 .
$$

Solving Eq. (46), we find

$$
\left|\phi_{1}\right|=5.48 \frac{\gamma_{l}^{2}}{\left(k_{\|} v_{t e}\right)^{2}}
$$


along with two spurious complex roots with negative real parts. Equation (4i) yields $\left|\phi_{1}\right|=0.87 \%$ for our simulation parameters, which is in good agreement with the the results shown in Figs. 1 and 4 . We have also tried an iterative solution using the linear value of $\delta f_{1}$ to obtain the first iteration $\delta f_{0}^{1}$, then using $\partial_{v \|} \delta f_{0}^{1}$ in Eq. (40) to predict $\delta f_{0}^{2}$. etc. However, this procedure did not converge.

\section{General Nonlinear Characteristic Method}

In this Section we extend the nonlinear scheme previously discussed for the electrostatic slab in Sec. 2 to the toroidal finite- $\beta$ gyrokinetic equations. We begin, as before, by writing $f(\mathbf{z}, t)=f_{0}(\mathbf{z})+\delta f(\mathbf{z}, t)$, where $\mathbf{z}=\left(\mathbf{R}, v_{\|}, \mu\right)$, and $f_{0}(\mathbf{z})$ is an equilibrium distribution which satisfies $\dot{\mathbf{z}}_{0} \cdot \dot{\partial}_{\mathbf{z}} f_{0}(\mathbf{z})=0$. Using the electromagnetic gyrokinetic equations with a nonuniform equilibrium B-field ${ }^{17-20}$ and writing $\dot{z}$ as an equilibrium and perturbed part, $\dot{\mathbf{z}}=\dot{\mathbf{z}}_{0}+\dot{\mathbf{z}}_{1}$, the equation for $\delta f$ is

$$
\partial_{t}\left(B^{*} \delta f\right)+\partial_{\mathbf{z}} \cdot\left(\dot{\mathbf{z}} B^{*} \delta f\right)=-\dot{\mathbf{z}}^{1} \cdot \partial_{\mathbf{z}} f_{0}
$$

where $\mu$ is time independent, and the equilibrium and perturbed trajectories are evolved using

$$
\begin{aligned}
\dot{\mathbf{R}}^{0} & =\frac{1}{B^{*}}\left\{v_{\|} \mathbf{B}^{* 0}+\frac{c}{e} \hat{\mathbf{b}} \times \mu \nabla B^{0}\right\}, \\
\dot{v}_{\|}^{0} & =-\frac{1}{B^{*}}\left\{\mathbf{B}^{u} \cdot \frac{\mu}{m} \nabla B^{0}\right\}, \\
\dot{\mathrm{R}}^{1} & =\frac{1}{B^{*}}\left\{v_{\|} \delta \mathbf{B}_{\perp}+\frac{c}{e} \hat{\mathbf{b}} \times \mu \nabla \delta B_{\|}+c \hat{\mathbf{b}} \times \nabla \delta \phi\right\}, \\
\dot{v}_{\|}^{1} & =-\frac{1}{B^{*}}\left\{\mathbf{B}^{* 0} \cdot\left(\frac{\mu}{m} \nabla \delta B_{\|}+\frac{e}{m} \nabla \delta \phi+\frac{e}{m c} \partial_{t} \delta A_{\|} \hat{\mathbf{b}}\right)+\delta \mathbf{B}_{\perp} \cdot \frac{\mu}{m} \nabla B^{0}\right\},
\end{aligned}
$$

where $\mathbf{B}^{*}=\mathbf{B}^{0}+\delta \mathbf{B}_{\perp}+\frac{m c}{e} u \nabla \times \hat{\mathbf{b}}, B^{*}=\hat{\mathbf{b}} \cdot \mathbf{B}^{*}$, and $\mathbf{B}^{* 0}=\mathbf{B}^{0}+\frac{m c}{e} u \nabla \times \hat{\mathbf{b}}$.

The characteristics (or particles) follow the full nonlinear trajectories $z=z^{0}+z^{1}$, and $\delta f$ is represented by

$$
B^{*} \delta f(\mathbf{z}, t)=\sum_{i} w_{i} \delta\left(\mathbf{z}-\mathbf{z}_{i}\right)
$$


We define $g$ as a smooth distribution function representing the particle distribution ( $g$ does not necessarily have to be equal to the physical distribution function $f$ at this point)

$$
B^{*} g(\mathbf{z}, t) \approx \sum_{i} \delta\left(\mathbf{z}-\mathbf{z}_{i}\right) .
$$

Substituting Eq. (53) into Eq. (48) and using Eq. (54), we obtain

$$
\dot{w}_{i}=-\left[\dot{\mathbf{z}}^{1} \cdot \frac{\partial_{\mathrm{z}} f_{0}}{g(\mathbf{z}, t)}\right]_{\mathrm{z}=\mathbf{z}_{1, t}} .
$$

which is just the generalization of Eq. (12). If , as before, we take $g=f=f_{0}+\delta f$ we obtain

$$
\dot{w}_{i}=-\left(1-w_{i}\right)\left[\dot{\boldsymbol{z}}^{1} \cdot \frac{\partial_{\mathbf{z}} f_{0}}{f_{0}}\right]_{\mathrm{z}=\mathbf{z}_{i}, t} .
$$

This evolution equation for $w_{i}$ along with the equations for the nonlinear trajectories Eqs. (49)-(52), is the more general version of the new method presented in Sec. 2.

\section{Discussion}

We have developed a new nonlinear characteristic method which retains all nonlinearities in a consistent way. This, however, does not preclude the possibility of neglecting various nonlinear terms if they are physically unimportant. In fact various terms can easily be "turned on and off" to test their physical effect. We also see no immediate difficulties in applying this method to other Vlasov-Maxwell systems where the derivatives of the initial distribution are known and finite. For a strong instability, where the perturbations become large $\delta f / f \approx 1$, noise properties revert back to those of a conventional particle simulation. However, in such a case the linear phase would be much more accurately resolved. At best, the new method captures the physics of conventional particle schemes with improved statistical properties. At worst, the scheme behaves linearly (with very low noise properties) for small perturbations, and fully nonlinear (with associated thermal noise) for large perturbations, and consistently makes the transition between the two extremes. We were able to obtain good energy conservation. However, compared to the number needed to capture the relevant physics of the drift wave model, a very large number of particles were required. The saturated electrostatic energy and associated change in electron kinetic 
energy is only $1.0 \%$ of the total electron thermal energy for the choice of parameters in Sec. 3. As sur ., it is not surprising that a relatively large number of particles, small timestep and fine grid where required to resolve this small change in kinetic energy $(1.0 \%$ of the total).

This one dimensional drift wave model permits us to isolate $E_{\|} \partial_{v_{\|}} \delta f$ nonlinearity and the associated nonlinear physics. Mode coupling theory was used to obtain a saturation level which is much lower (for our choice of parameters) than the estimate based on the energy balance calculation of Sagdeev and Galeev. ${ }^{5}$ Sirnulation results agree well with our estimate. Because of this new lower saturation amplitude, parallel velocity nonlinearity may play a more important role in microturbulence then previously thought, although past investigations have shown such a tendency. ${ }^{11}$ In addition, the existing linear theory for tokamak geometry predicts a ballooning type mode structure which is elongated in the radial direction and, therefore, will reduce the effectiveness of the $E \times B$ nonlinearity for saturation.

Finally, the nonlinear characteristic method was extended to the general electromagnetic gyrokinetic equations. Application of these equations in a three dimensional toroidal simulation is an ongoing effort and will be reported in the future. ${ }^{15}$

\section{Acknowledgments}

We thank Dr. Liu Chen, Dr. T.S. Hahm, and Dr. A. Dimits for enlightening discussions. This research was supported in part by an appointment to the U.S. Department of Energy Fusion Energy Postdoctoral Research Program administered by Oak Ridge Associated Universities and by DOE Contract No. DE-AC02-76-CHO-3073. 


\section{References}

${ }^{1}$ A. Limits and W. W. Lee, PPPL Report 2718, Oct. 1990 [submitted to J. Comp. Phys.].

${ }^{2}$ T. Tajima and F.W. Perkins, Proceedings of the Sherwood Fusion Theory Conference, 2P9 (1983).

${ }^{3} \mathrm{M}$. Kotschenruether. Proceedings of the 14 th International Conference on Vumerical Simulation of Plasmas (Anapolis. MD 1991).

${ }^{4}$ W.W. Lee, J. Comput. Phys. 72, 243 (1987).

${ }^{5}$ R.Z. Sagdeev and A.A. Galeev, Nonlinear Plasma Theory, W.A. Benjamin, Inc., NY (1969).

${ }^{6}$ J.A. Byers, Proceedings of the Fourth Conference on Numerical Simulation of Plasmas (NRL,Washington, DC: 1970), P. 496.

'J.P. Friedberg, R.L. Morse, and C.W. Nielson, Proceedings of the Third Conference on Numerical Simulation of Plasmas (Stanford University, CA 1969).

${ }^{8}$ J. Denavit and J.M. Walsh, Comments in Plasma Phys. Controlled Fusion 6 209 (1981).

${ }^{9}$ B.I. Cohen, S.P. Auerbach, J.A. Byers, and H. Weitzner, Phys. Fluids 23. 2529 (1980).

${ }^{10}$ A. Friedman, R.N. Sudan, and J. Denavit, J. Comput. Phys. $401(1980)$.

${ }^{11}$ W.W. Lee. J.A. Krommes, C.R. Oberman, and R.A. Smith, Phys. Fluids 272652 (1984).

${ }^{12}$ W. M. Nevins, Phys. Fluids 22, 1681 (1979).

${ }^{13}$ S.C. Cowley, R.M. Kulsrud and R. Sudan, Phys. Fluids B 132767 (1991).

${ }^{14}$ Romenelli. L. Chen and S. Briguglio, Phys. Fluids B 32496 (1984).

${ }^{15}$ S.E. Parker and W.W. Lee, Proceedings of the International Sherwood Fusion Theory Conference, 1B3 (1992).

${ }^{16}$ W.W. Lee, W.M. Tang, Phys. Fluids, 31612 (1988). 
${ }^{17}$ T.S. Hahm, W.W. Lee and A. J. Brizard. Phys. Fluids 311940 (1988).

${ }^{18}$ T.S. Hahm, Phys. Fluids 312670 (1988).

${ }^{19}$ A. J. Brizard, J. Plasma Physics 41541 (1989).

${ }^{20}$ A.J. Brizard, Ph.D. Thesis, Princeton Univ., Jan. 1990.

\section{Figures}

Figure 1 (a) Time history for the real (solid line) and imaginary (dashed line) part of the electrostatic potential for the $n=1 \mathrm{drift}$ instability $\left(k_{\perp} \rho_{i} \simeq 0.8\right)$, and (b) the corresponding amplitude evolution for the run with 987 particles on a 16 -grid system.

Figure 2 (a) Perturbed distribution $\delta f(k=0) / f_{0}\left(v_{\|}=0\right)$, and (b) the total distribution $f / f_{0}\left(v_{\|}=0\right)$ for the electrons for the 987 particle run at $\Omega_{i} t=500$.

Figure 3 (a) Time history for the electron particle flux (solid line), and the time rate of change for the electron parallel momentum (dashed line), and (b) the time evolution for the perturbed electron kinetic energy (solid line), and the field energy (dashed line) for the 987 particl? run.

Figure 4 (a) Time history for the real (solid line) and imaginary (dashed line) part of the electrostatic potential for the $m=1$ drift instability $\left(k_{\perp} \rho_{i} \simeq 0.8\right)$, and (b) the corresponding amplitude evolution for the run with 46368 particles on a 64-grid system.

Figure 5 (a) Perturbed distribution $\delta f(k=0) / f_{0}\left(v_{\|}=0\right)$, and (b) the total distribution $f / f_{0}\left(v_{\|}=0\right)$ for the electrons for the 46368 particle run at $\Omega_{i} t=500$.

Figure 6 (a) Time history for the electron particle flux (solid line), and the time rate of change for the electron parallel momentum (dashed line), and (b) the time evolution for the perturbed electron kinetic energy (solid line), and the field energy (dashed line) for the 46368 particle run. 

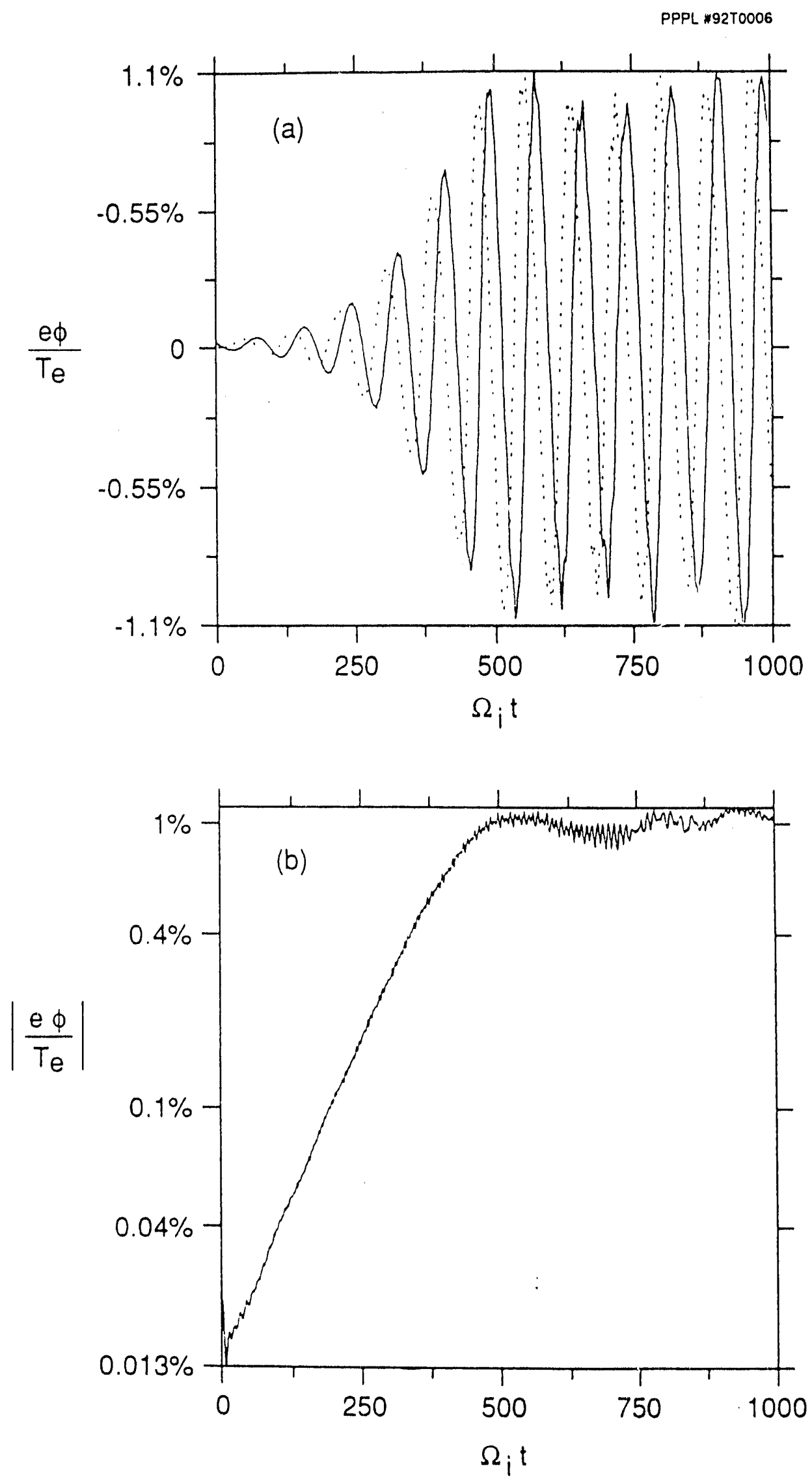

Fig. 1 

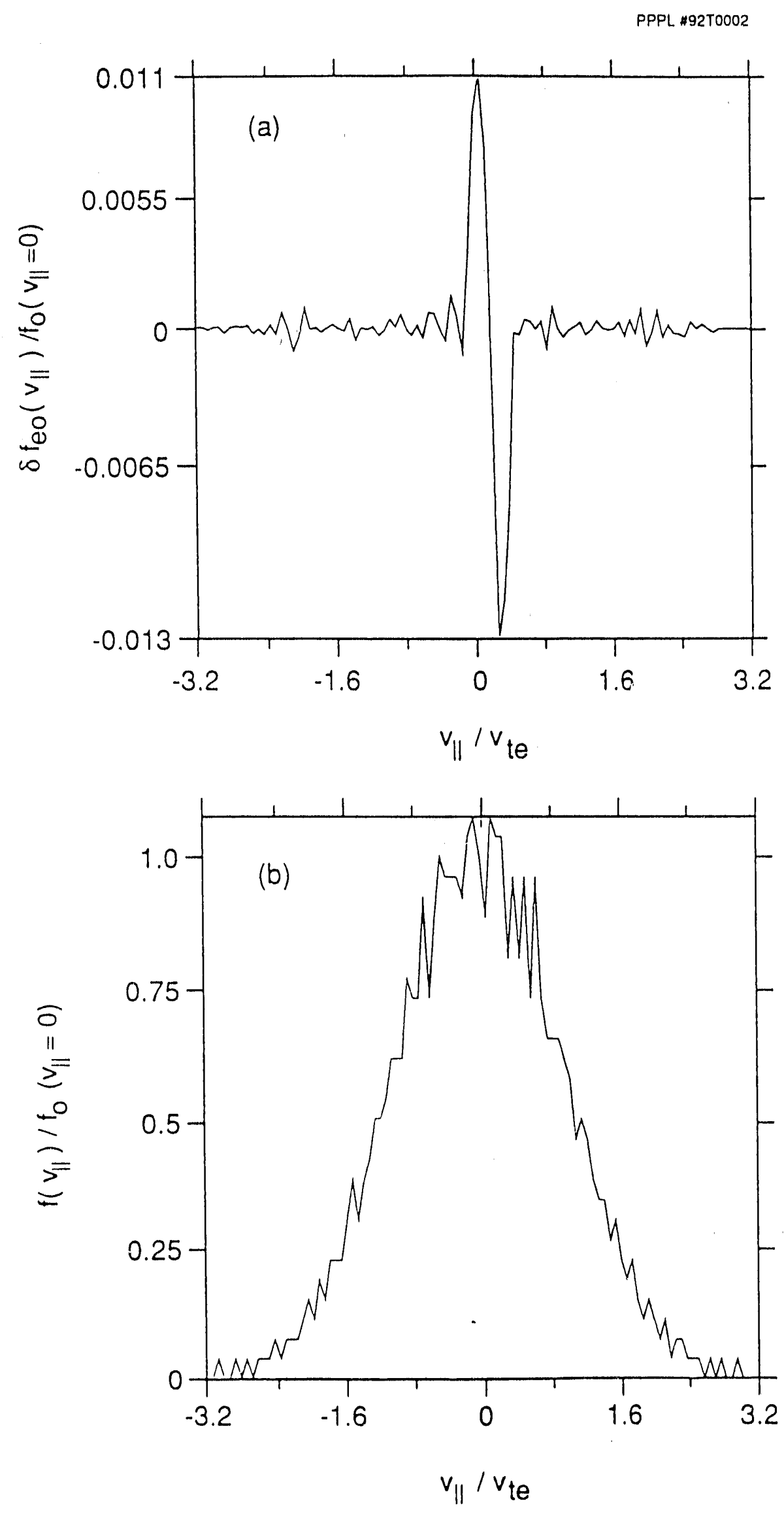

Fig. 2 

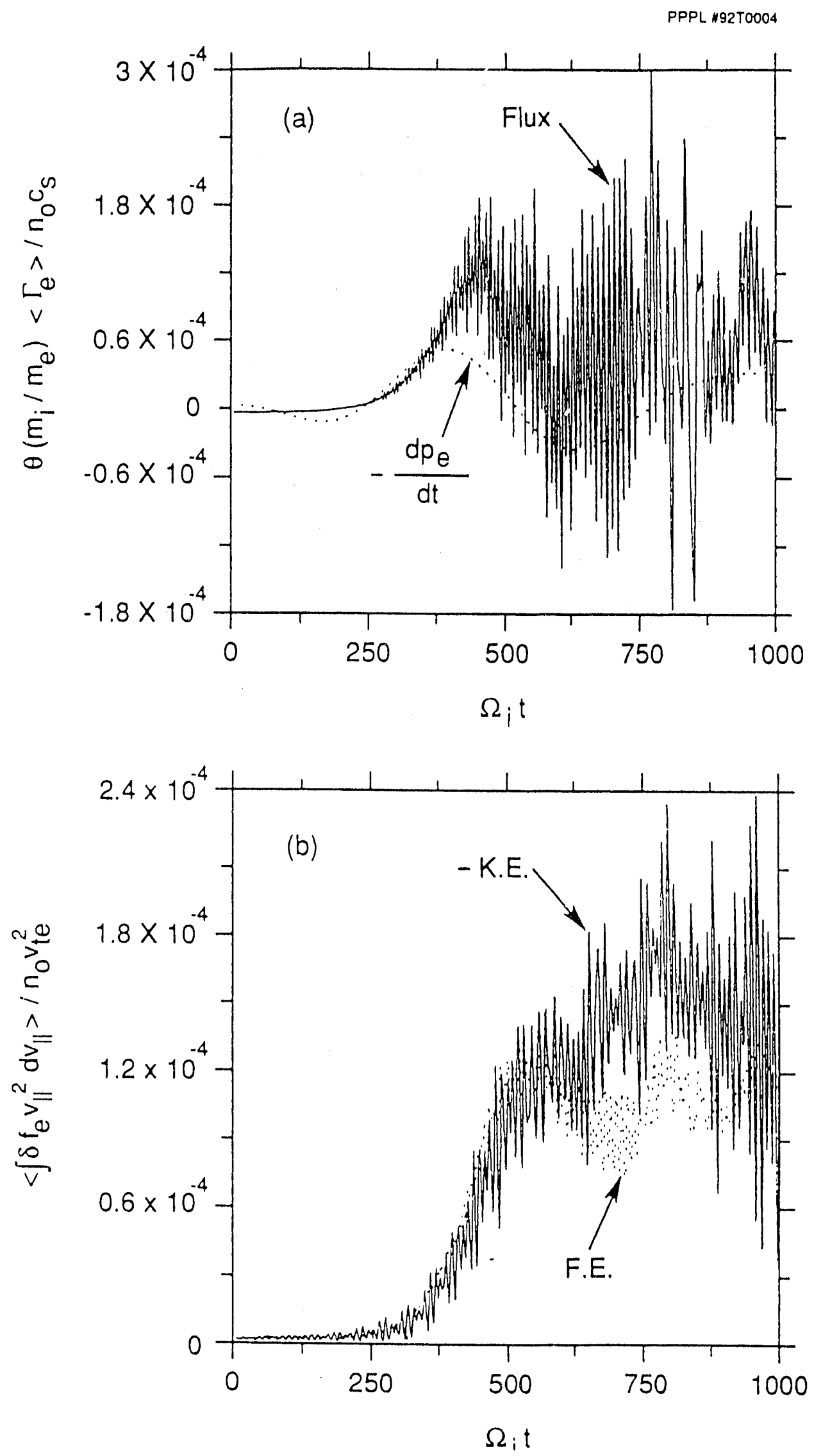

Fig. 3 

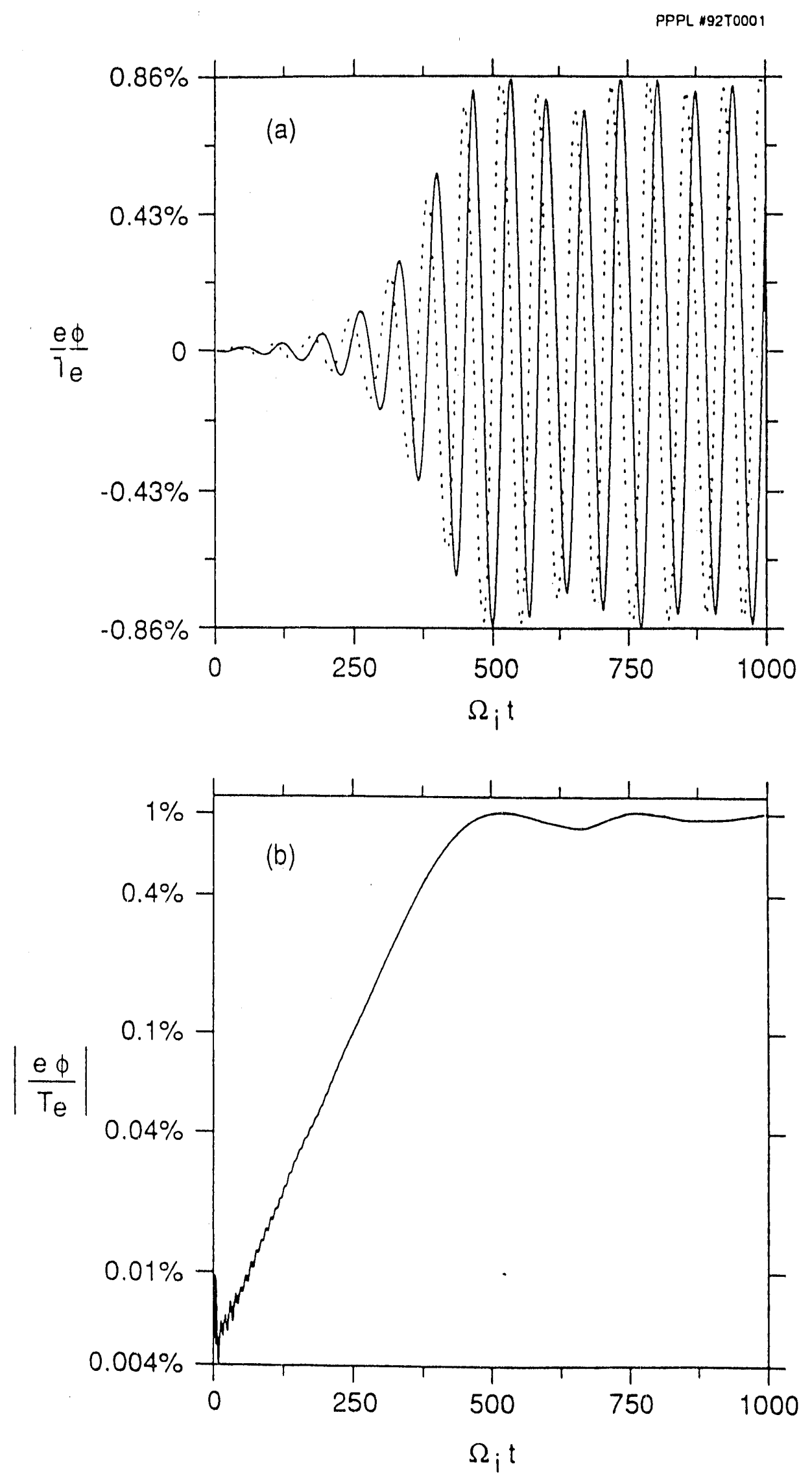

Fig. 4 

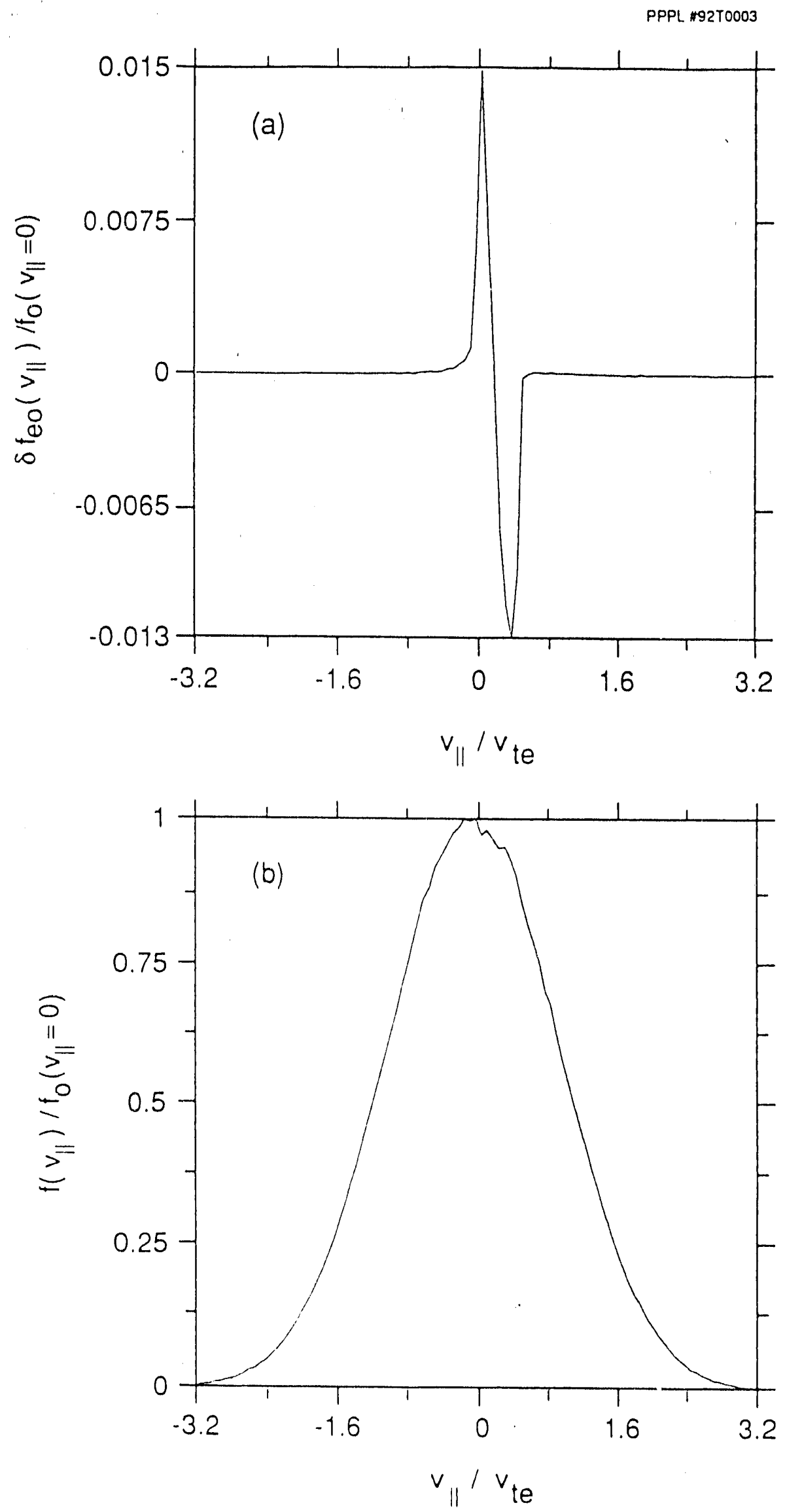

Fig. 5 

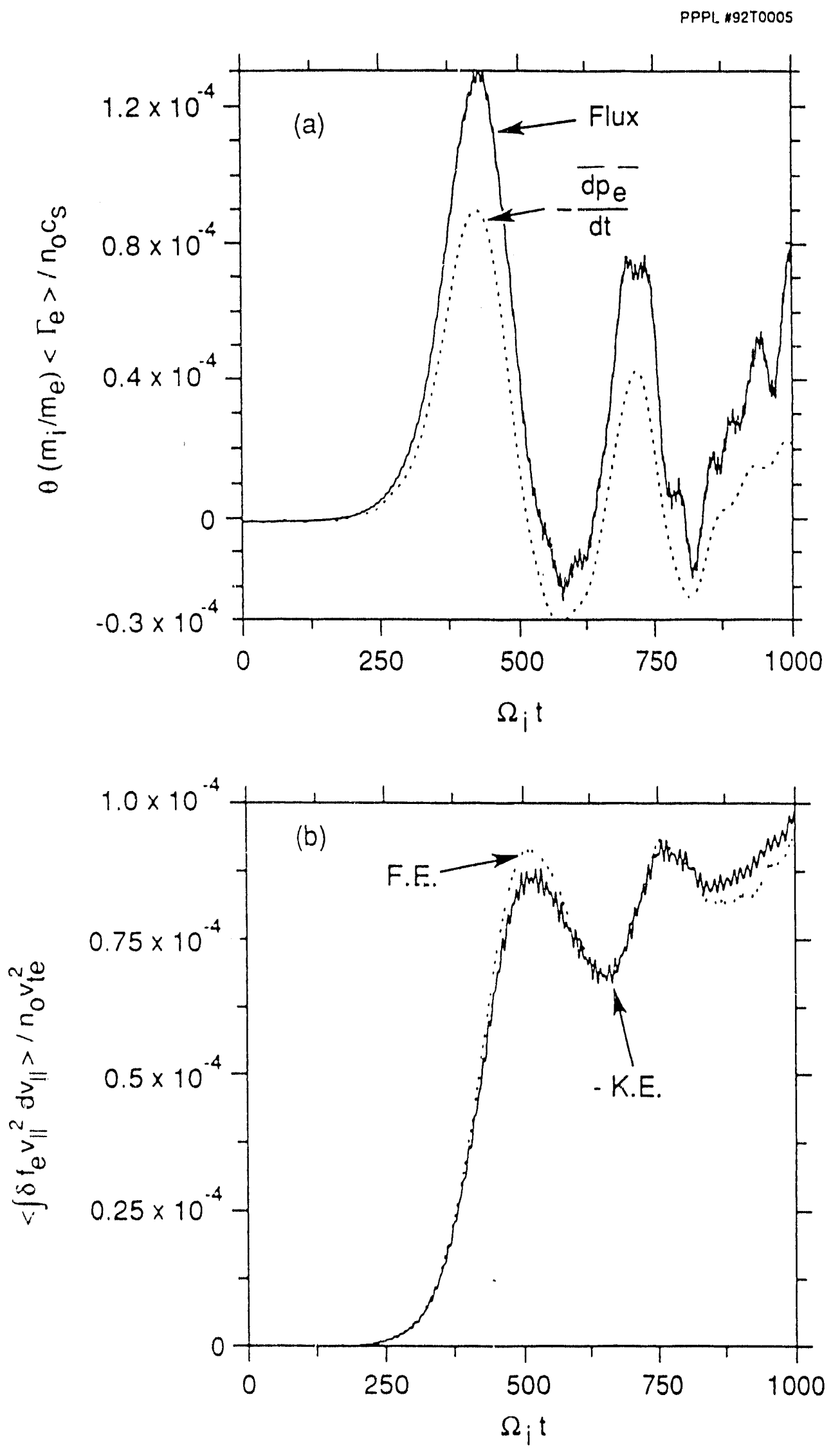

Fig. 6 
Dr. F. Peoloni, Univ. of Wollongong, AUSTRALIA

Prot. M.H. Brennan, Univ. of Syoney, AUSTRALIA

Plasma Research Lob., Australien Nat. Univ., AUSTRALIA

Prof. I.R. Jones, Flinders Univ, AUSTRALIA

Prof. F, Cap, inst for Theoretical Physics, AUSTRIA

Prof. M. Hoinder, Instiut for Theoretische Physik, AUSTRIA

Prof. M. Goossons, Astronomisch Instituut, BELGIUM

Ecolo Royde Mitivire, Leb. do Phy. Plasmas, BELGIUM

Commission-Europeen, DG. Xli-Fusion Prog., BELGIUM

Prof. R. Bouciqub, Rijkeunivenibit Gent, BELGIUM

Dr. P.H. Sakanaka, Instituto Fisica, BRUZIL

Instituto Neciond Do Peequisas Especiavi-INPE, BRUZIL

Documents Orioe, Alomic Energy of Ceneda Ld., CANADA

Dr. M.P. Bectyneki, MPB Technolopies, Inc., CANADA

Dr. H.M. Skaregard, Univ. of Sackatchewen, CANADA

Prof. J. Toichmarn, Univ. of Montreal, CANADA

Prof. S.R. Sreenivaen, Univ. of Celogry, CANADA

Prof. T.W. Johnston, INRS-Energie, CANADA

Dr. R. Botion, Contre canedien de fusion megnétique, CANADA

Dr. C.R. James, Univ. of Aberta, CANADA

Dr. P. Lukbe, Komencketho Universzita. CZECHOSLOVAKIA

The Librarian, Cutham Luboralory, ENGLAND

Librey, R61, Authertord Appleton Laboratory, ENGLAND

Mrs. S.A. Hutchinson, JET Library, ENGLAND

Dr. S.C. Sherme, Univ. of South Procific, FIJI ISLANDS

P. Menonen, Univ. of Helsinki, FINLAND

Prof. M.N. Buseace, Ecolo Polytechnique, FRANCE

C. Nourstor, Lo de Physique des Milioux lonises, FRANCE

J. Radot, CENCADARACHE - Bat 506, FRANCE

Prof. E. Economou, Univ. of Crote, GREECE

Ms. C. Rinni, Univ. of loamina, GREECE

Dr. T. Mud, Acadomy Bibliographic Ser., HONG KONG

Preprint Librery. Hungarian Academy of Sa., HUNGARY

Dr. B. DesGupa, Saha inst of Nucteer Ptycics, INDIA

Dr. P. Kaw, Inst. for Plasma Recearch, INDIA

D. P. Rocensu, lereed Inst of Tectinology, ISPAEL

Librerien, Intemationd Conber for Theo Phyeics. ITALY

Miss C. Do Palo, Associaziono EURATOMENEA, ITALY

Dr. G. Groseo, Istituto de Fisica del Plasma, ITALY

Prof. G. Rostangni, Istitus Gas lonizzati Del Cnr, ITALY

Dr. H. Yemeto, Toehiba Pas a Dower Conm, JAPAN
Prof. I. Kewakemi, Hiroshima Univ., JAPAN

Prot. K. Nishikawa, Hiroshima Univ., JAPAN

Director, Jepen Atomic Energy Research Inst, JAPAN

Prot. S. Ioh, Kyushu Univ., JAPAN

Research Into. Ct., National Instit. for Fusion Science, JAPS. i

Prof. S. Tenmen, Kyoto Univ., JAPAN

Librey, Kyoto Univ., JAPAN

Prot. N. Inowe. Uniry. of Tolyyo, LAPAN

Secretary, Plema Section, Electrotectinical Lab., JAPAN

S. Mori, Techniced Acticor, JAEPI, MAPAN

Dr. O. Mined, Kumenos Inst of Technology. JAPAN

J. Hyoon-Sook, Koren Abonic Enorgy Roseurch Inst, KOREA

D.I. Chai, The Koree Adv. Inst of Sa. \& Tech., KOREA

Prof. B.S. Liley, Univ. of Weikato, NEW ZEALAND

Inst of Phyics, Chinves Aand SCI PEOPLE'S REP. OF CHINA

Librey, Inst of Plasma Physics, PEOPLE'S REP. OF CHINA

Tsinchua Univ. Librey, PEOPLE'S REPUBLIC OF CHINA

Z. L. S.W. Int Phycics, PEOPLE'S REPUBUC OF CHINA

Prof. J.A.C. Cebrd, Instiuto Superior Tecnico, PORTUGAL

Dr. O. Potrus, al I cuza Univ., Romania

Dr. J. de Villiare, Fucion Studies, AEC, S. AFRICA

Prof. M.A. Hewborg. Univ. of Natal, S. AFRICA

Prot. D.E. Kim, Pohang Inst. of Sci. \& Tech., SO. KOAEA

Prof. C.I.E.M. A.T, Fusion Division Library, SPAIN

Dr. L Samfo, Univ. of UNEA, SWEDEN

Librery, Royd inet. of Technology, SWEDEN

Prol. H. Whatmeon, Chelmers Univ. of Tech., SWEDEIN

Centre Phys. Des Plammes, Ecole Potrtech, SWITZERLAND

Bibliotheak, Inst. Voor Plasma-Fysica, THE NETHERLANDS

Asst. Prot. Dr. S. Cetir, Midde East Tech. Univ., TURKEY

Dr. V.A. Gukhilh,Sci. Res. Inst. Electrophys.I Apparatus, USSA

Dr. D.D. Austov, Siberian Brench of Academy of Sci., USSR

Dr. G.A. Eiseov, I.V. Kurcintov Inst, USSA

Librerien, The Ukr.SSA Academy of Scionces, USSR

Dr. LM. Kovizhmykh, Inst. of General Physics, USSR

Kerntorechungeunlege GmbH, Zentrabibliothek, W. GERMANY

Bibliothok, Inst. For Plasmatorschung, W. GERMANY

Prof. K. Schinder, Ruhr-Univereity Bochum, W. GERMANY

Dr. F. Wegner, (ASOEX), Max-Planck-Institut, W. GERMANY

Librerien, Max-Plenck-Institur, W. GEAMANY

Prot. R.K. Jenev, Inat of Physice, YUCOSLAVIA 

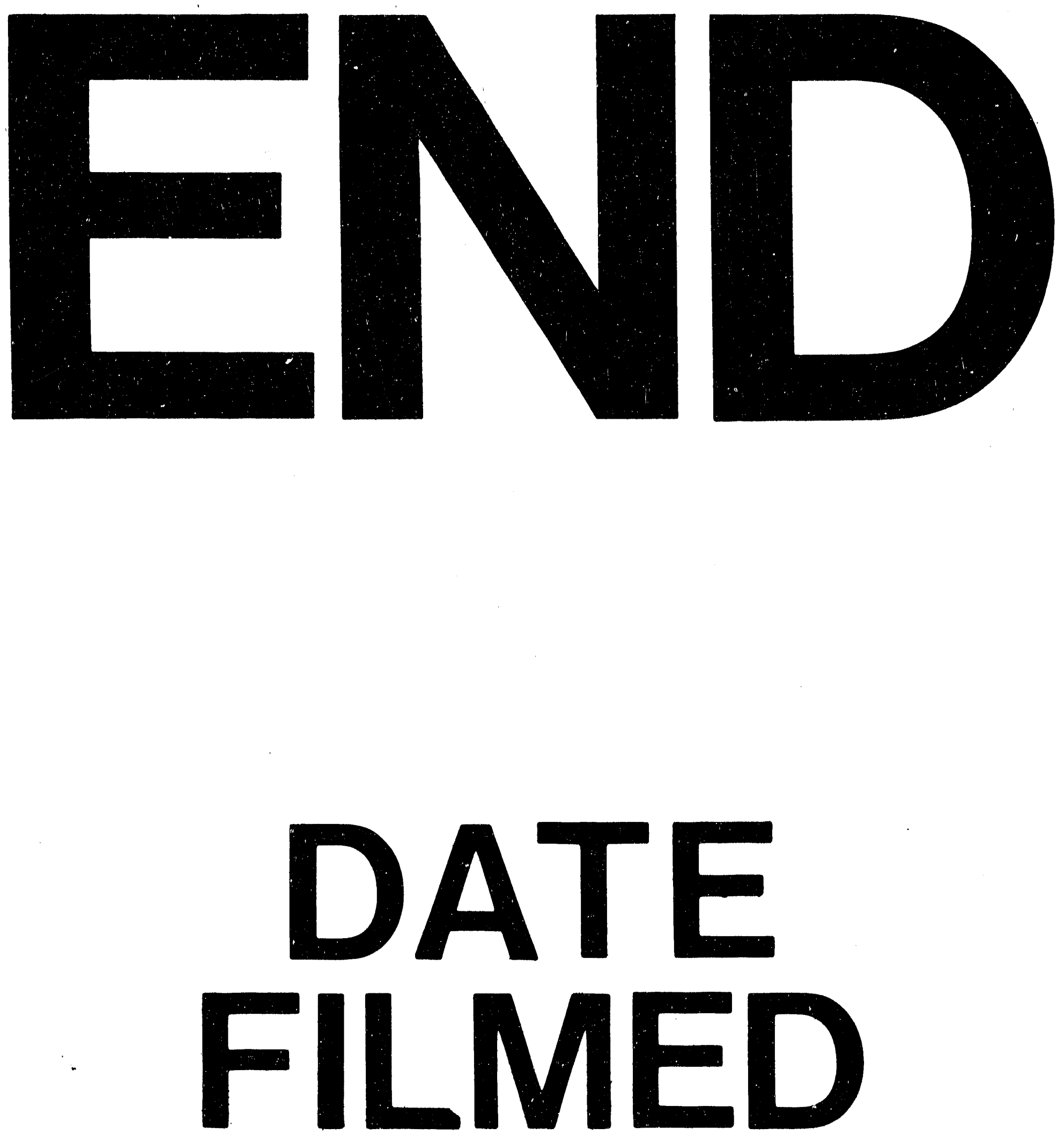

I

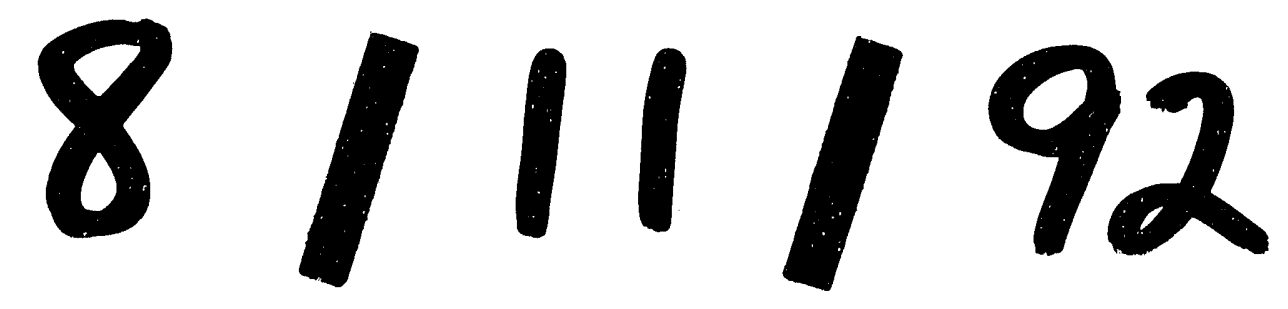


